\title{
A NOTE ON THE LARGEST REGULAR SUBALGEBRA OF A BANACH ALGEBRA
}

\author{
JYUNJI INOUE AND SIN-EI TAKAHASI
}

(Communicated by Paul S. Muhly)

\begin{abstract}
A simple proof of Albrecht's result on the existence of the largest closed regular subalgebra of a semisimple commutative Banach algebra is given.
\end{abstract}

Albrecht [1] proved that any semisimple commutative Banach algebra with identity has the largest closed regular subalgebra, using the theory of decomposable operators. In this short note, we give a simple proof of Albrecht's result without the assumption of semisimplicity. Our proof is quite different from Albrecht's, and based on the consideration of the hull-kernel topology of the carrier space of the given Banach algebra.

Theorem. If $A$ is a commutative Banach algebra with identity, then there exists a closed regular subalgebra of $A$ which contains all closed regular subalgebras of $A$.

Our proof is essentially based on the following

Lemma. Let $X$ be a commutative Banach algebra with identity and $B$ a Banach subalgebra of $X$. If $B$ is regular, then for any $b \in B$ the Gelfand transform of $b$ as an element of $X$ is continuous on the carrier space $\Phi_{X}$ of $X$ in the hull-kernel topology.

Proof. We can assume without loss of generality that $B$ contains the identity of $X$. Then it is sufficient to show that the restriction map $\theta: \Phi_{X} \rightarrow \Phi_{B}$; $\varphi \rightarrow \varphi \mid B$ is continuous in the hull-kernel topology. To do this let $F$ be a closed subset of $\Phi_{B}$ in the hull-kernel topology. Then $\left\{\varphi \in \Phi_{X}: \varphi \mid \operatorname{ker} F=0\right\}=$ $\theta^{-1}(F)$. Also since $\operatorname{ker} F \subset \operatorname{ker} \theta^{-1}(F)$, it follows that hul $\left(\operatorname{ker} \theta^{-1}(F)\right) \subset\{\varphi \in$ $\left.\Phi_{X}: \varphi \mid \operatorname{ker} F=0\right\}$. Therefore $\theta^{-1}(F)$ is closed in the hull-kernel topology. In other words, $\theta$ is continuous in this topology.

Proof of Theorem. Let $\operatorname{reg}(A)$ be the closed subalgebra of $A$ generated by the class of all closed regular subalgebras of $A$. It is sufficient to show that $\operatorname{reg}(A)$ is regular. Note that $\operatorname{reg}(A)$ is a commutative Banach algebra with identity. If

Received by the editors November 29, 1989.

1980 Mathematics Subject Classification (1985 Revision). Primary 46H05.

Key words and phrases. Commutative Banach algebra, regular, hull-kernel topology.

Supported in part by a Grant-in-Aid for Scientific Research from the Japanese Ministry of Education. 
$b$ is an element of $A$ which belongs to a certain regular Banach subalgebra $B$ of $A$, then $B \subset \operatorname{reg}(A)$. Hence by the preceding lemma the Gelfand transform of $b$ as an element of $\operatorname{reg}(A)$ is continuous on $\Phi_{\operatorname{reg}(A)}$ in the hull-kernel topology. Since $\operatorname{reg}(A)$ is generated by such $b$ 's, the Gelfand transform of an arbitrary element of $\operatorname{reg}(A)$ is also continuous on $\Phi_{\operatorname{reg}(A)}$ in the hull-kernel topology. Consequently $\operatorname{reg}(A)$ is regular.

\section{REFERENCES}

1. E. Albrecht, Decomposable systems of operators in harmonic analysis, Toeplitz Centennial (I. Gohberg, ed.), Birkhäuser, Basel, 1982, pp. 19-35.

Department of Mathematics, Hokkaido University, Sapporo 060, Japan

Department of Basic Technology, Yamagata University, Yonezawa 992, Japan 